\title{
Evidencias de dominio incaico en la región de Atacama: Hacia una sistematización de la ocupación de la cuenca del río Jorquera ${ }^{1}$
}

Nelson GAETE G. ${ }^{2}$

\section{RESUMEN}

Este trabajo corresponde a nuestra primera mirada, sobre el tipo de ocupación y dominio incaico en la cuenca del río Jorquera, formativa del río Copiapó, y pone su acento en organizar un cuerpo de "viejos" y "nuevos" datos en forma sistemática.

\begin{abstract}
This work corresponds to our first look, on the occupation type and domain incaico in the basin of the Jorquera river, formative of the Copiapó river, and it puts its accent in organizing a body of "old" and "new" data in systematic form.
\end{abstract}

\section{Antecedentes generales}

Nuestro interés sobre el Inca, está dado por intentar sistematizar e incorporar al conocimiento de la arqueología y prehistoria de la región de Atacama, un importante cuerpo de viejos y nuevos "datos" acerca de la ocupación y explotación inca en la cuenca del río Jorquera (Iribarren 1958), una de las subcuencas formativas del Copiapó. Parte de estos "nuevos" datos, han sido descritos o redescritos en años recientes por Carlos Ocampo, Andrea Seelenfreund, Miguel Cervellino y Nelson Gaete, como parte de trabajos ambientales sobre

1 Este artículo es parte del trabajo "Asentamiento Incaico en la Cuenca del río Jorquera, Provincia de Copiapó, Región de Atacama", desarrollado en el Seminario Problemas de Prehistoria Andina, Magister en Arqueología, Universidad de Chile

22 Oriente $\mathrm{N}^{\circ}$ 634, Talca. Email: negaete@entelchile.net el componente patrimonio cultural realizados para empresas mineras de la zona, información en gran parte inédita contenida en informes, manuscritos y diarios de terreno (Ocampo 1994 Ms, Seelenfreund 1997 Ms, Cervellino y Gaete 1997a Ms y 1997b, Cervellino et al. 1997a Ms, 1997b Ms y 1998 Ms, Gaete y Cervellino 1998 Ms, Gaete et al. 1997a Ms, 1997b Ms, 1997c Ms, 1997d Ms y $1998 \mathrm{Ms})$.

Hemos puesto el énfasis en buscar indicadores que puedan manifestar al Inca en el área objeto del estudio. Básicamente, éstos se refieren a rasgos estructurales o constructivos que refieren tradicionalmente lo incaico (Kendall 1974), como por ejemplo, el registrar la presencia de RPC (recinto perimetral compuesto), en las instalaciones (Madrazo y Otonello 1966, Raffino 1981). Sin embargo, una variable que consideramos por ahora más segura, es el registro contextual de materiales culturales muebles, como es el caso de presencia de alfarería Inca, Diaguita-Inca, e IncaCopiapó, las que en nuestro caso funcionan como categoría diagnóstica (Gaete 1999 Ms).

Nuestro método de trabajo, en esta primera mirada a la forma de dominio y ocupación del Inca de la cuenca del Jorquera, pone su acento en organizar la información en un cuerpo de datos coherente. Es por ello, que la parte medular del trabajo está comprendido por la descripción de los contextos, así como su organización en bases de datos.

La información contextual en algunos casos es bastante rica, sobre todo para aquellos sitios que hoy cuentan con excavaciones sistemáticas. En 
otros, es del todo fragmentaria, pero es lo que hay. $\mathrm{Al}$ respecto, no hemos querido forzarla, por lo que este trabajo manifiesta el estado actual de la investigación en la cuenca del Jorquera. Dentro de este marco, hemos considerado la información édita, principalmente el valioso trabajo de don Hans Niemeyer (1986), sobre la ocupación incaica de la cuenca alta del río Copiapó.

\section{Algunos elementos sobre el área que nos ocupa}

La cuenca del río Copiapó,

"se desarrolla entre las latitudes $26^{\circ} 45^{\prime}$ y $28^{\circ} 30^{\prime}$ $S$, en el centro de la III región de Atacama... El rio Copiapó se forma en La Junta, a $1.230 \mathrm{~m}$, de la confluencia de sus dos más importantes tributarios, el río Jorquera que viene del norte y el río Pulido que proviene del sur oriente... Pocos kilómetros más debajo de La Junta cae al Copiapó el río Manflas que proviene del sur" (Niemeyer 1986).

Son estos tres ríos los formativos del río Copiapó.

La cuenca del río Jorquera

"drena una superficie de la cordillera andina de aproximadamente $4.160 \mathrm{~km}^{2}$, situada al nordoriente y al oriente de la cuenca del río Copiapó; y al occidente y al sur de la cordillera de Darwin. Esta cadena montañosa es la que separa los últimos relictos, los más australes, de un área altiplánica con cuencas endorreicas (como la del Salar de Maricunga y la de la laguna del Negro Francisco), de los tributarios de ríos con desagüe al Pacífico" (Niemeyer et al. 1998).

El río Jorquera tiene su origen en la confluencia del río Figueroa con la quebrada Vizcachas del Jorquera ( $2.550 \mathrm{~m} . \mathrm{snm})$, en el sector de Las Banderitas. Entre este punto, y la quebrada Castaños (curso superior), presenta sectores de terrazas aluviales de buen desarrollo, las que en parte hasta el día de hoy son utilizadas como campos de cultivo por los actuales habitantes del valle.

El río Figueroa,

"viene directamente del norte y recoge las aguas del flanco poniente de la cordillera Darwin. Trans- curre en un valle ancho relleno de sedimentos que se interrumpe a veces por angosturas" (Niemeyer 1986).

Este, se origina en el flanco poniente del Volcán Azufre o Copiapó, el que forma parte de la cordillera Darwin. Al Figueroa desagua el río Aguas Blancas y la quebrada de Paredones como afluentes principales. Manifiesta un amplio desarrollo de zonas de vegas en la caja del río, las cuales son prácticamente un continuo desde el sector de Las Banderitas, hasta la zona de desagüe de la quebrada de Paredones.

En el sector de La Guardia, confluye al Figueroa el río Turbio, el que nace de la confluencia de los ríos de Los Piuquenes y Cachitos, en el sector de la Junta del Cachitos, a 3.130 m.snm. En general, el río Turbio discurre por un cajón muy estrecho, el cual presenta a ambos lados un alto farellón rocoso que lo encierra. Lo anterior, posibilita solo estrechas terrazas, las cuales se alternan a uno u otro lado del río, con una escasa vegetación asociada al curso de agua mismo.

El río Cachitos, se origina de la confluencia del arroyo Peña Negra y la quebrada El Pan (3.670 $\mathrm{m} . \mathrm{snm}$ ), originándose el primero, a los pies del paso de Peña Negra en la divisoria de aguas de la Cordillera de Los Andes. El otro formativo del Turbio, es el río de Los Piuquenes, el que muestra un amplio y sostenido desarrollo de vegas en toda su extensión, y el cual se origina de la confluencia de los ríos Nevado y Pircas Negras. El río Nevado, tiene su origen en el Cordón Yeguas Heladas en el sector donde se emplaza el Volcán Jotabeche, y tiene como principales afluentes al tío de La Gallina y al de Quebrada Seca. Presenta en su curso inferior, un amplio sector de llanos (Llano del Nevado y Llano Pastillos), los cuales muestran un amplio desarrollo de vegas, o más bien algo parecido a bofedales salinos. El otro formativo del Nevado, es el río Pircas Negras, el cual tiene su origen en el estero Comecaballos, que nace a los pies del portezuelo de Comecaballos, en la divisoria de aguas de la cordillera andina.

Climáticamente. la cuenca del Jorquera se inscribe dentro del desierto frío de montaña (parte inferior y media), y el clima de tundra de alta montaña (parte superior), y en cuanto a unidades natu- 


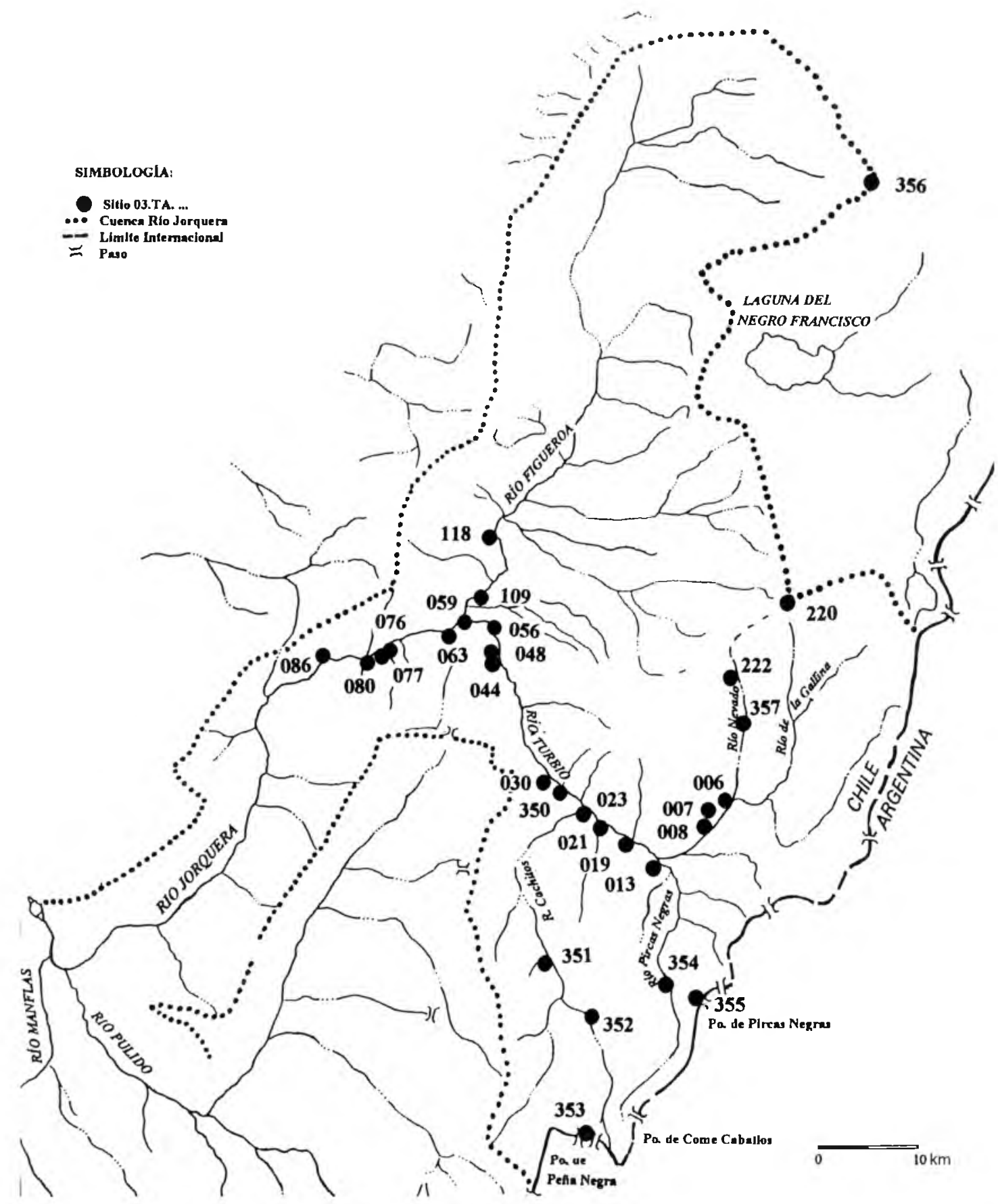

Figura 1. Plano de ubicación de los sitios con manifestación inca (cuenca río Jorquera, región de Atacama) 
rales, dentro del Subsistema Preandino de la Cuenca del Copiapó en su parte inferior y media, como del Subsistema Altiplánico de Diego de Almagro en su parte superior (I.G.M. 1989).

La cuenca del Jorquera posee un conjunto de pisos altitudinales complementarios, lo que posibilita su ocupación durante todo el año, con ambientes propicios para la agricultura en su porción inferior (valle del río Jorquera), así como para la ganadería en su parte media y superior. A grandes rasgos, el área posee un tipo de paisaje que guarda evidentes relaciones ambientales con lo que génericamente conocemos como "puna" o "puna salada", área que además actúa como límite y zona de contacto o transición, entre lo que llamamos "Norte Chico" y el área altiplánica o de los salares.

\section{El Inca en la cuenca del río Jorquera}

La información que hemos logrado recopilar (Gaete $1999 \mathrm{Ms}$ ), se presenta a continuación de dos maneras diferentes. Por una parte, la totalidad de la información disponible, tanto édita como inédita, se ha organizado en bases de datos, donde la Base de Datos 1 contiene los elementos de identificación y localización; la Base de Datos 2, refiere la situación de emplazamiento del sitio, las formas de las estructuras observadas, así como las asociaciones que el sitio pueda manifestar; la Base de Datos 3, nos muestra aquella parte mueble del contexto, tanto a nivel de artefactos como de ecofactos. Por otra parte, se describe un conjunto de "nuevos" sitios incas, los cuales son una muestra de los diferentes tipos de instalaciones que se observan en la cuenca del Jorquera (Figura 1).

\section{Sitio 03 TA 086 "El Castaño"}

El sitio, ya conocido y descrito con anterioridad (Iribarren 1958, Seelenfreund 1997 Ms), se emplaza en la primera y segunda terraza del río Jorquera, en un sector donde la quebrada Castaños confluye a éste. El extenso e importante sitio arqueológico manifiesta, a lo menos tres asentamientos, dados por un "poblado" de la Cultura Copiapó, emplazado en el extremo oeste de la segunda terraza; una instalación Inca, que puede corresponder a un gran centro administrativo emplazado en el área norte y noroeste de la segunda terraza; y un asentamiento habitacional Copiapó-
Inca-Diaguita, localizado en el área noroeste en la primera terraza (Cervellino et al. $1998 \mathrm{Ms}$, Cervellino y Gaete 1999). A continuación, describiremos los dos últimos, que son los que manifiestan presencia incaica.

\section{Poblado Copiapó-Inca}

La excavación realizada por nosotros en este sector (Cervellino et al. $1998 \mathrm{Ms}$ ), mostró que el yacimiento corresponde a parte de un asentamiento habitacional Copiapó-Inca-Diaguita, propia del Período Tardío, y del cual fue posible observar parte de una estructura habitacional con muros pircados, la que muestra dos unidades o recintos adosados de formas tendientes a circulares. Hacia el exterior de los recintos, se registran densos basureros que caen hacia cotas inferiores, los que en parte vienen a actuar como un reforzamiento de los muros pircados. En un tiempo posterior, este poblado habría sido "tapado" por basuras provenientes desde las grandes estructuras incaicas situadas unos pocos metros más arriba.

Los materiales exhumados, muestran los elementos propios de la Cultura Copiapó, en clara asociación contextual con otros de clara filiación Inca y Diaguita-Inca. Es así, por ejemplo, que los tipos cerámicos Copiapó y Punta Brava, partes de formas como pucos, platos, y grandes vasijas continentes, se encuentran asociados a alfarería inca, partes de vasijas con formas de aríbalos, escudillas playas, y vasijas con pedestal, así como a cerámica Diaguita-Inca, partes de platos y pucos de paredes rectas, todos los cuales manifiestan formas y decoración característica, la cual es ampliamente conocida.

\section{Instalación Inca}

Este asentamiento, que con cierta certeza puede ser caracterizado como un importante centro administrativo incaico, se caracteriza por un conjunto compuesto por tres grandes estructuras de patrón arquitectónico del tipo recinto perimetral compuesto (RPC), de los cuales hoy sólo se conservan sus basamentos, en parte tapados por los sedimentos de los muros de barro destruidos.

El RPC 1 se emplaza en el área norte de la segunda terraza, en la parte más alta de ésta, a $27 \mathrm{~m}$ al 
oeste de la quebrada Castaños, y muy cerca del borde de la terraza, con dimensiones observables de $104 \mathrm{~m}$ de largo y entre 20 y $30 \mathrm{~m}$ de ancho. Parte del RPC, hacia su lado norte, se encuentra tapado por el talud del camino actual que asciende por la quebrada, por lo que su ancho total hoy no es posible de observar. El RPC 2 se emplaza en el área noroeste de la segunda terraza, unos 95 $\mathrm{m}$ al suroeste del RPC 1, con dimensiones observables de $18 \mathrm{~m}$ de largo y $10 \mathrm{~m}$ de ancho. EI RPC 3 se emplaza en el área noroeste de la segunda terraza, unos $51 \mathrm{~m}$ al suroeste del RPC 2, con dimensiones observables de $23 \mathrm{~m}$ de largo y $11 \mathrm{~m}$ de ancho. Cabe destacar, además, la existencia de los restos de un muro perimetral, emplazado en el borde de la terraza y que une el RPC 2 con el RPC 3 , con una extensión aproximada a los $51 \mathrm{~m}$ de largo.

Seelenfreund (1997 Ms), al realizar trabajos de conservación preventiva, refiere el registro de sectores de enterratorios humanos, dejados al descubierto por excavaciones ilegales.

Tanto en superficie, como en sectores del depósito expuesto por huaqueo, se observa fragmenteria cerámica de los tipos Copiapó, Punta Brava, Diaguita-Inca, e Inca.

Además, hasta hoy se registran partes de un antiguo camino en el sector, el que podría corresponder a parte del Camino del Inca.

\section{Sitio 03 TA 080 "La Aduana"}

Corresponde a un tambo incaico, el cual está formado por una estructura de $20 \mathrm{~m}$ de largo y $4 \mathrm{~m}$ de ancho, dividido internamente en cuatro recintos contiguos, en un patrón del tipo recinto perimetral compuesto (RPC). Presenta muros dobles con relleno y argamasa. En superficie muestra abundante material lítico y cerámico. Se observan partes de otras estructuras, las que se encuentran destruidas por reocupación histórica (Cervellino et al. 1997a Ms).

El sitio, en superficie, muestra gran cantidad de fragmentería cerámica, principalmente del tipo alisado monocromo, así como escasos fragmentos del tipo Copiapó (variedad negro sobre rojo), asociada al componente arqueológico.
En la excavación, se registraron escasos fragmentos cerámicos, donde destacan los pulidos negros, café, y gris-café. El material lítico se resume al registro de una lasca en andesita. El vegetal, a abundantes restos de carrizo y algunas semillas (Gaete et al. 1997b Ms).

\section{Sitio 03 TA 076 "Tambo de Pailahuén"}

Corresponde a un tambo incaico, formado por dos estructuras distante unos $12 \mathrm{~m}$ una de otra, la más pequeña de 3 por $5 \mathrm{~m}$ y la mayor de 12 por $11 \mathrm{~m}$, esta última conteniendo tres recintos menores en su interior, con patrón del tipo Recinto Perimetral Compuesto (RPC). Los muros son de piedra, dobles con relleno y argamasa. Se encuentra ubicado en el plano inclinado formado por la desembocadura de una quebrada al río Jorquera. En superficie se registra cerámica alisada monócroma y fragmentos de tipo Diaguita-Inca, así como material lítico dado por manos de moler, lascas y desechos en jaspe, cuarzo y calcedonia (Cervellino et al. 1997a Ms).

Este tambo, además, muestra clara asociación con un asentamiento habitacional de población Copiapó (03 TA 077 "Los Fósiles"), el cual se emplaza a unos $70 \mathrm{~m}$ hacia el oeste, y que en su parte baja exhibe enterratorios humanos con cerámica Copiapó, Punta Brava y Diaguita-Inca (Gaete et al. 1997b Ms, Gaete y Cervellino 2000).

\section{Sitio 03 TA 056 "Tambo del Turbio"}

El sitio se encuentra emplazado sobre la terraza norte del río Turbio, ocupando una extensión de unos $100 \mathrm{~m}$ de largo (eje E/O) y $30 \mathrm{~m}$ de ancho (eje N/S), entre el talud del farellón rocoso y el margen norte del río, en un sector muy estrecho.

De acuerdo con Seelenfreund (1997 Ms), en el sitio

"destaca una plataforma de piedra de planta rectangular de $4 \times 4$ metros y $70 \mathrm{~cm}$ de alto sobre la cual se construyó una cúpula de piedra y barro que hoy se encuentra colapsada....corresponde probablemente a un horno de actividades mineras de data colonial".

Asimismo, menciona que 
"se observan varios recintos muy derrumbados en los alrededores; otros en mejor estado de conservación fueron reocupados por pastores. Los muros son gruesos y levemente inclinados hacia adentro, formados por dos hileras de piedras grandes y argamasa con piedras pequeñas rellenando los espacios intermedios. Las esquinas internas están bien delimitadas... Las estructuras se ordenan en torno a una zona despejada de piedras (plaza) de unos 20 metros de largo por 15 metros de ancho. Hacia el norte de esta plaza se encuentra la estructura "horno" y hacia el sur de ella se ubican varios recintos, entre ellos uno de grandes dimensiones (10 15 metros) muy destruido."

En fecha posterior, nosotros inspeccionamos este sitio (Cervellino et al. 1997b Ms), logrando observar que está compuesto por un conjunto de recintos dispersos, todos de planta rectangular, con muros de piedra en técnica de doble muro con relleno y argamasa. En superficie, registramos material lítico y cerámico. En el lítico, una punta de proyectil de forma triangular con pedúnculo y aletas, de tamaño pequeña, tipo Copiapó, así como desechos de talla y algunas lascas en cuarzo y calcedonia. En la cerámica, fragmentos de vasijas alisadas monocromas, dos fragmentos del tipo Copiapó variedad negro sobre rojo, un fragmento que podría corresponder a parte de una vasija Diaguita-Inca, así como fragmentería cerámica alisada no diagnóstica.

Al respecto, podría tratarse de un asentamiento incaico, probablemente un tambo, dado el patrón constructivo y la disposición espacial que tienen las estructuras, el cual es reocupado en tiempos históricos.

\section{Sitio 03 TA 030 "Tambería del Río Turbio"}

El sitio, se encuentra emplazado en la confluencia de la quebrada La Tambería con el río Turbio, sobre parte del cono de deyección, inmediato a una zona de vegas ribereñas.

De acuerdo al informe elaborado por Seelenfreund (1997 Ms), el sitio es de época Inca-Diaguita, y está formado por tres estructuras aisladas unas de otras:

"Estructura A: Recinto rectangular dividido in- ternamente en dos recintos casi cuadrados, cuyos muros están muy derrumbados. Uno de los recintos tiene un vano de acceso orientado al SE. La estructura tiene 5 metros de largo por 2,5 metros de ancho. Los recintos miden en el interior $2 \times 2$ metros. No se registra material arqueológico en superficie."

"Estructura B: Ubicada a $40 \mathrm{~m}$ al $\mathrm{NW}$ de la estructura $C$ se compone de tres recintos cuadrangulares de $3 \times 3$ metros adosados unos a otros y dispuestos en hilera a un costado de un recinto rectangular abierto de mayor tamaño. Este último a su vez presenta una división interior. No se registra material arqueológico en superficie."

"Estructura C: Formada por tres recintos dispuestos en línea al costado $N E$ de un recinto rectangular abierto, del tipo de un recinto perimetral compuesto $(R P C)$ de patrón inca. Dos de los recintos adosados a la plaza mayor miden $2 \times 3$, el tercero está muy destruido y sus dimensiones no se aprecian claramente. Sobre estos recintos se han construido dos estructuras con muros de piedra y techo de dos aguas para uso de los arrieros. Adosados a las estructuras modernas hay recintos rectangulares más pequeños de data actual." "Los muros originales están construidos con piedras rectangulares seleccionadas y el espacio entre las hileras de piedra está relleno con piedras más pequeñas y barro. Miden hasta 1 metro de ancho en su base... En superficie sólo se encontraron dos fragmentos de cerámica engobada rojo exterior e interior sin engobe sobre una pasta naranja muy bien cocida..."

Con posteridad, se logró registrar en superficie algunos fragmentos cerámicos del tipo Copiapó, variedad negro sobre rojo, así como un fragmento de tipo Diaguita-Inca (Cervellino et al. 1997 b Ms).

\section{Sitio 03 TA 019 "Corral de Yañez"}

El sitio, se encuentra emplazado en el borde sur de la vega del río de Los Piuquenes (Llano Vega de Piuquenes), en el sector del Llano Corral de Yañez, adosado al farellón rocoso que limita la caja del río, ocupando un área de unos $200 \mathrm{~m}$ de largo en eje E/O y $20 \mathrm{~m}$ de ancho en eje N/S.

Se compone, a lo menos, de cuatro grandes es- 
tructuras pircadas, dos de las cuales son del tipo recinto perimetral compuesto, propio del patrón constructivo inca. La tercera estructura, presenta dos recintos de planta circular adosados a un bloque pétreo aislado. La cuarta, registra un recinto de planta rectangular de grandes proporciones, el que presenta adosado otro más pequeño en uno de sus costados. Gran parte de los muros son del tipo doble muro con relleno, los que en sus bases alcanzan al metro de espesor. Es probable, que el sitio fuera mayor y que parte de él haya sido destruido por el camino actual que lo atraviesa. Presenta evidencias de reutilización subactual como corrales, por lo que la superficie registra una gruesa capa de guano (Gaete et al.1997d Ms).

Cabe destacar, que en una revisita al sitio fue posible examinarlo con más detención, registrando una punta de proyectil "diaguita", de forma triangular con aletas y pedúnculo central, elaborada en cuarzo, así como un fragmento cerámico del tipo Copiapó variedad negro sobre rojo, y otro Diaguita-Incaico.

\section{Sitio 03 TA 013 "Pircas Negras"}

El sitio, probablemente un asentamiento aldeano de uso estacional, se encuentra localizado en el área de confluencia de los ríos Nevado y Pircas Negras que forman el río de Los Piuquenes, emplazándose sobre la ladera y parte superior de la terraza sur del río Pircas Negras, y ocupando un área de unos $80 \mathrm{~m}$ de largo en eje E/O y $45 \mathrm{~m}$ de ancho en eje N/S. En la caja del río, se desarrolla una extensa zona de vegas, denominadas Llano Vegas de Piuquenes.

Corresponde a un sitio habitacional, compuesto por a lo menos unas 15 estructuras aisladas, cada una de ellas conformada por dos recintos de planta tendientes a circular, con medidas variables entre 3 a 4 m de diámetro, para el recinto mayor, y 1,2 a $1,8 \mathrm{~m}$ de diámetro para el recinto menor. Muestran muros tipo pirca seca, los cuales hoy se encuentran fuertemente colapsados. Al menos una de las estructuras, la emplazada más cerca de la vega, presenta reutilización actual por pastores.

En superficie, se registra escaso material cultural, dado por derivados de núcleo sin modificación (lascas y desechos de talla), elaborados en calce- donia, jaspe y basalto, así como material cerámico que corresponde a tres fragmentos de platos Copiapó, en su variedad negro sobre rojo, y un fragmento de una vasija Diaguita-Inca (Gaete $e t$ al. $1997 \mathrm{c} \mathrm{Ms).}$

\section{Sitio 03 TA 007 "Tambería del Río Nevado"}

El sitio, probablemente un asentamiento aldeano de uso estacional, se encuentra emplazado sobre la terraza fluvial oeste del río Nevado, a unos 20 $\mathrm{m}$ sobre el lecho del río, mirando hacia el Llano del Nevado, en el área de confluencia de los ríos Nevado y de La Gallina, en un sector donde desaguan dos quebradas que caen al río, siendo una de ellas la quebrada Las Tamberías.

De acuerdo con Niemeyer (1986), quién realizó el levantamiento del sitio y lo registró por vez primera, éste se compone de 36 estructuras que denomina "recintos", los que se encuentran dispersos en un área de $150 \mathrm{~m}$ de largo y $80 \mathrm{~m}$ de ancho máximo, registrándose "aislados, aglutinados en parejas, o bien aglutinados de a tres, siendo el tercero del grupo como una dependencia menor", y en donde

"la planta predominante de los recintos es la circular o la ligeramente elíptica con diámetros variables de 1,40 m para los más pequeños hasta de 7,70 m para los más grandes."

Para este sitio, Ocampo, observa

"alrededor de 24 estructuras de pirca compuestas de recintos de forma predominantemente subcuadrangular que tienen promedio de $4 \mathrm{~m}$ de ancho por $6 \mathrm{~m}$ de largo. Algunos poseen recintos circulares más pequeños de aproximadamente 2 $m$ de diámetro, adosado a un costado compartiendo uno de los muros" (1994 Ms).

De acuerdo a nuestra observación (Gaete et al. $1997 \mathrm{c} \mathrm{Ms}$ ), las estructuras conformadas por muros elaborados con cantos rodados, poseen formas tendientes a circulares y ovaladas, principalmente, y en donde las diferencias de apreciación. pueden estar dadas por el mal estado de conservación de los muros, que en gran parte se registran colapsados. 
El sitio muestra escaso material cultural en superficie, principalmente lítico, ya que en gran parte se encuentra tapado por acumulaciones de arenas más recientes, siendo el registro más abundante en sectores que evidencian excavaciones ilegales (huaqueo), donde es posible apreciar un denso depósito cultural conformado por cenizas, material lítico y cerámico, así como abundantes restos óseos de camélidos, los que se ubican tanto al interior como al exterior de las estructuras. El material lítico, registra derivados de núcleo sin modificación (lascas, láminas, y desechos de talla), núcleos, preformas de instrumentos, y una punta de proyectil de tamaño pequeño, todos elaborados en cuarzo, calcedonia, jaspe. cuarcita, basalto, andesita, y obsidiana. El material cerámico, fragmentos de vasijas correspondientes a formas utilitarias monócromas, platos Copiapó (variedad negro sobre rojo), vaso campaniforme y platos Diaguita-Inca, y escudillas playas Inca (Niemeyer 1986, Ocampo 1994 Ms, Gaete et al. 1997c Ms).

\section{Sitio 03 TA 006 "Llano del Nevado"}

Corresponde a un sitio habitacional, probablemente una aldea de uso estacional, que ocupa un área de unos $50 \mathrm{~m}$ de largo y $25 \mathrm{~m}$ de ancho, compuesto a lo menos por ocho estructuras pircadas de planta circular y diámetro variable entre los 4 y 6 $\mathrm{m}$, las que presentan uno o dos recintos más pequeños adosados a sus muros. Se emplaza a ambos lados de una quebrada que cae hacia el río Nevado, inmediatamente al Suroeste de la Quebrada Las Tamberías. En superficie, se registra material cultural lítico y cerámico diverso, donde destaca el registro de dos fragmentos cerámicos del tipo Copiapó variedad negro sobre rojo, y un fragmento de plato Diaguita-Inca (Gaete et al. 1997c Ms).

\section{Sitio 03 TA 357 "Maranceles 2"}

De acuerdo a Ocampo (1994 Ms), el sitio se encuentra emplazado sobre un faldeo de la ladera oeste del cajón del río Nevado, entre dos pequeñas quebradas que corren perpendiculares al río, y asociado a sectores de vegas. Corresponde a una estructura pircada de planta tendiente a rectangular, con dimensiones de $6 \mathrm{~m}$ de largo máximo, en sentido $\mathrm{N}-\mathrm{S}$ (interior $4,5 \mathrm{~m}$ ), y ancho máximo de $3,6 \mathrm{~m}$ en sentido $\mathrm{E}-\mathrm{O}$ (interior $2 \mathrm{~m}$ ). Los muros, presentan un espesor promedio de $0,7 \mathrm{~m}$ y un alto máximo de $1,1 \mathrm{~m}$.

En superficie, se aprecia gran cantidad de material lítico, disperso en un radio cinco veces superior al perímetro de la estructura, elaborado sobre diversas materias primas tales como jaspe, cuarzo, calcedonia, sílices, basaltos y obsidiana. Los artefactos corresponden mayoritariamente a lascas primarias bifaciales desechadas, con retoques bimarginales, trozos de cortex, núcleos agotados y desechos de talla. También fue posible registrar instrumentos, como varios raspadores laterales y tipo "uña", cuchillos bifaciales, tres puntas de proyectil fracturadas elaboradas en jaspe y calcedonia. A la entrada del recinto mayor, se registró una piedra de mortero confeccionada en roca andesítica. El material cerámico, corresponde a fragmentos de vasijas de tipo utilitario monocromo color café, en su mayoría; sin embargo, también se recolectaron fragmentos del tipo Copiapó variedad negro sobre rojo, y cerámica Inca.

Para Ocampo,

"se trata de un interesante sitio habitacional con una larga secuencia ocupacional en el periodo agroalfarero, que involucra los periodos temprano, medio y tardio, con ocupaciones tipo Molle, Copiapó e Incaica" (1994 Ms).

\section{Sitio 03 TA 222 "Tambo del Río Nevado"}

Se emplaza en la terraza oeste del río Nevado, en un sector donde la primera terraza penetra hacia la ladera del cerro, formando una especie de pequeña rinconada. Se trata de un tambo incaico, que ocupa un área de unos $50 \mathrm{~m}$ de largo y $30 \mathrm{~m}$ de ancho, el cual probablemente se encuentre asociado a la plataforma inca ceremonial ubicada en la cima del Nevado Jotabeche que describe Reinhard (1991).

Corresponde a un sitio donde es posible observar al menos tres estructuras con muros de piedra. La estructura 1, es un RPC de planta tendiente a rectangular, con dimensiones aproximadas de $20 \mathrm{~m}$ de largo y $10 \mathrm{~m}$ de ancho, y que en su interior se encuentra dividido en seis recintos, el mayor de unos $12 \mathrm{~m}$ de largo y $10 \mathrm{~m}$ de ancho, encontrándose en su pared sur el vano de acceso. Registra en sus vértices NO y SO dos recintos de planta 
tendiente a circular adosados a la pared perimetral. La estructura 2, también corresponde a un RPC de planta tendiente a rectangular, con dimensiones aproximadas de $10 \times 10 \mathrm{~m}$ de lado, y dividido en tres recintos interiores. La estructura 3, mucho más pequeña, corresponde a un recinto de planta tendiente a circular, la que en parte se encuentra tapada por sedimentos arenosos (Gaete y Cervellino $1998 \mathrm{Ms}$ ).

\section{Algunos comentarios}

De la información que hemos logrado sistematizar, puede llevarse a cabo un primer nivel de análisis, acerca de la ocupación y explotación inca de la cuenca del Jorquera.

Es así, que hemos logrado sistematizar la información disponible para 29 sitios, los cuales muestran ciertas tendencias de cómo se estructura en el espacio esta ocupación.

De los 29 sitios reseñados, 22 de ellos se localizan en sectores de confluencia y/o angosturas, lo que puede manifestar que estas instalaciones tienen por función controlar los flujos de entrada y salida, tanto de personas como de bienes en el territorio que se domina. A lo anterior, debemos agregar que al menos 13 sitios manifiestan claramente asociación con evidencias que refieren a senderos o vías de circulación antiguas, las que podrían ser relictos del Camino del Inca.

El registro del sitio 03 TA 086 El Castaño reafirma lo anterior, dado que es posible pensar en la cuenca del Jorquera como una unidad con características propias, y en donde el dominio y control se ejerce desde esta importante instalación. Desde esta perspectiva, cabría la posibilidad que cada uno de los formativos del río Copiapó, así como el valle mismo, tuvieran por parte del Inca una administración diferenciada, de acuerdo a un mejor manejo tanto de la población como de los recursos explotados en los diferentes ambientes que conforman este territorio.

En relación con la producción o explotación de recursos, los sitios documentan bastante bien un manejo diferenciado con respecto a actividades agrícolas y ganaderas. Es así como los asentamientos ubicados en el río Jorquera. manifiestan asociación con campos de cultivo y obras de riego de los mismos, a diferencia de los asentamientos de los ríos Figueroa, de los Piuquenes, Cachitos, Nevado y Pircas Negras, que se relacionan principalmente con el pastoreo de camélidos.

Por otra parte, si bien no tenemos información directa acerca de la actividad minera, podemos pensar que esta labor se está llevando a cabo fuer-

\begin{tabular}{|c|c|c|c|c|c|}
\hline $\begin{array}{c}\text { MUESTRA } \\
\text { No LAB. }^{\circ} \text { LAB. }\end{array}$ & $\begin{array}{c}\text { PROCEDEN- } \\
\text { CIA }\end{array}$ & $\begin{array}{c}\text { FRAGMENTO } \\
\text { FECHADO }\end{array}$ & $\begin{array}{c}\text { EDAD } \\
(\mathbf{A} \tilde{N} O S \text { AP) }\end{array}$ & $\begin{array}{c}\text { FECHA (CON } 2 \\
\text { SIGMAS) }\end{array}$ & FECHA TL \\
\hline UCTL-1181 & $\begin{array}{l}03 \text { TA } 086 \\
\text { Cuadr: J- } 4 \\
\text { Nivel: } 50-60 \mathrm{~cm}\end{array}$ & $\begin{array}{l}\text { Copiapó, } \\
\text { Negro sobre } \\
\text { Rojo }\end{array}$ & $520 \pm 55$ & 1420 a $1530 \mathrm{DC}$ & $1475 \mathrm{DC}$ \\
\hline UCTL- 1182 & $\begin{array}{l}03 \text { TA } 086 \\
\text { Cuadr: J-4 } \\
\text { Nivel: } 50-60 \mathrm{~cm}\end{array}$ & Inca & $450 \pm 50$ & 1495 a $1595 \mathrm{DC}$ & $1545 \mathrm{DC}$ \\
\hline UCTL- 1180 & $\begin{array}{l}03 \text { TA } 080 \\
\text { Rec. } 2 \text {, Cuadr. } 1 \\
\text { Nivel: } 10-20 \mathrm{~cm}\end{array}$ & $\begin{array}{l}\text { Café } \\
\text { Pulido }\end{array}$ & $445 \pm 45$ & 1505 a 1595 DC & $1550 \mathrm{DC}$ \\
\hline UCTL-1289 & $\begin{array}{l}03 \text { TA } 109 \\
\text { Estructura } 2 \\
\text { Nivel: } 45-55 \mathrm{~cm}\end{array}$ & Inca & $440 \pm 50$ & 1510 a $1610 \mathrm{DC}$ & $1560 \mathrm{DC}$ \\
\hline
\end{tabular}


temente durante el Tardío, ya que la cuenca del Jorquera dispone de una amplia gama de recursos minerales (cobre, plata, y oro), lo que guarda una evidente relación con las numerosas explotaciones actuales.

Con respecto a la cronología, contamos por vez primera para esta área con fechados absolutos (TL) para el Período Tardío. los cuales fueron procesados por el laboratorio de termoluminiscencia de la Universidad Católica.

Antes de terminar, con esta primera aproximación a los datos, debemos hacer mención a algunos elementos propios que muestra la cuenca del Jorquera.

Durante el Período Intermedio Tardío, la cuenca del Jorquera se encuentra fuertemente ocupada por una población que hoy denominamos Cultura Copiapó (Niemeyer et al. 1998), y cuyo rasgo arqueológico distintivo es la alfarería Copiapó y Punta Brava. Estos grupos, que practicaban un modo de producción basado en la agricultura, ganadería y minería, no muestran en sus contextos habitacionales materiales exógenos, tales como alfarería Diaguita, la cual ingresa con el Inca a la cuenca del Jorquera, lo que hace suponer que es éste último quien traslada a esta población hacia la tercera región de Atacama. Llama la atención, asimismo, que en los contextos Copiapó tardíos se continúan manifestando los mismos tipos alfareros del Intermedio Tardío, sin mostrar cambios significativos, pero ahora "mezclados" con to Diaguita e Inca (Gaete y Cervellino 1997 y 1999).

Finalmente, y como una manera de recapitular en esta primera mirada, pensamos que el espacio comprendido en la cuenca del Jorquera manifiesta claramente la ocupación y dominio de esta área por parte del Inca, información relevante a la hora de construir o reconstruir modelos regionales al respecto. En esta perspectiva, el área registra un importante centro administrativo, puestos de controf, tambos, y dos santuarios de altura, los que reafirman un marcado dominio.

\section{BIBLIOGRAFIA}

CERVELlino, M. y N. GAETE. Estudio Proyecto camino 1997 Ms a de acceso desde Copiapó a Mina Refugio/Compañía Minera Maricunga. Tramo Río Figueroa. Línea de Base Patrimonio Cultural. Comuna de Tierra Amarilla, Provincia de Copiapó. III Región de Atacama, para Compañía Minera Maricunga.

1997 Ms b Estudio Proyecto camino de acceso desde Copiapó a Mina Refugio/Compañía Minera Maricunga. Tramo Quebrada Carrizalillo. Línea de Base Patrimonio Cultural. Comuna de Tierra Amarilla, Provincia de Copiapó, III Región de Atacama, para Compañía Minera Maricunga.

1999 Asentamientos del Período Intermedio Tardío y del Tardío en el sitio El Castaño, río Jorquera, valle de Copiapó-Chile. Actas Primeras Jornadas de Arqueología de La Rioja-Relaciones Trasandinas. La Rioja, Argentina. (en prensa).

CERVELlino, M., N. GAETE e I. MARTINEZ. Estudio 1997 Ms a Proyecto camino de acceso desde Copiapó a Mina Refugio/Compañía
Minera Maricunga. Tramo Río Jorquera. Línea de Base Patrimonio Cultural. Comuna de Tierra Amarilla, Provincia de Copiapó, III Región de Atacama, para Compañía Minera Maricunga.

1997 Ms b Estudio camino de acceso desde La Guardia a Proyecto Aldebarán. Tramo Río Turbio. Línea Base Patrimonio Cultural. Comuna de Tierra Amarilla, Provincia de Copiapó, III Región de Atacama, para Compañía Minera Cerro Casale (Placer Dome Latin America).

$1998 \mathrm{Ms} \quad$ Proyecto camino de acceso desde Copiapó a Mina Refugio/Compañía Minera Maricunga. Trabajos Intervención Arqueológica del Sitio 03 TA 086 El Castaño. Comuna de Tierra Amarilla, Provincia de Copiapó, III Región de Atacama, para Compañía Minera Maricunga.

GAETE, N. Asentamiento incaico en la cuenca del 1999 Ms río Jorquera, Provincia de Copiapó, Región de Atacama. Trabajo final Seminario Problemas de prehistoria andina, Magister en arqueología, Universidad de Chile, Santiago. 
GAETE, N. y M. CERVELLINO. Asentamientos habitaciona2000 les de la Cultura Copiapó en el río Jorquera, formativo del río Copiapó región de Atacama, Chile. Actas del XIV Congreso Nacional de Arqueología Chilena, Contribución Arqueológica 5, Tomo II: 607-629, Copiapó.

1998 Ms Estudio áreas de prospección minera Proyecto Aldebarán. Línea Base Patrimonio Cultural. Comuna de Tierra Amarilla, Provincia de Copiapó, III Región de Atacama, para Compañía Minera Cerro Casale (Placer Dome Latin America).

1999

Sitios habitacionales de la Cultura Copiapó en el río Jorquera, valle de Copiapó, Región de Atacama. Chile. Actas Primeras Jornadas de Arqueología de La Rioja-Relaciones Trasandinas. La Rioja, Argentina, (en prensa).

GAETE, N., M. CERVELlino, e I. MARTINEZ. Proyecto 1997 Ms a camino de acceso desde Copiapó a Mina Refugio/Compañía Minera Maricunga. Tramo Río Figueroa. Trabajos de Mitigación de Impactos y de Conservación Preventivas. Comuna de Tierra Amarilla, Provincia de Copiapó, III Región de Atacama, para Compañía Minera Maricunga.

1997 Ms b Proyecto camino de acceso desde Copiapó a Mina Refugio/Compañía Minera Maricunga. Tramo Río Jorquera. Trabajos de Mitigación de Impactos y de Conservación Preventivas. Comuna de Tierra Amarilla, Provincia de Copiapó, III Región de Atacama, para Compañía Minera Maricunga.

1997 Ms c Estudio camino de acceso desde La Guardia a Proyecto Aldebarán. Tramo Río Nevado. Línea Base Patrimonio Cultural. Comuna de Tierra Amarilla, Provincia de Copiapó, III Región de Atacama, para Compañía Minera Cerro Casale (Placer Dome Latin America)

1997 Ms d Estudio camino de acceso desde La Guardia a Proyecto Aldebarán. Tramo Río Piuquenes. Línea Base Patrimonio Cultural. Comuna de Tierra Amarilla, Provincia de Copiapó, III Región de Atacama, para Compañía Minera Cerro Casale (Placer Dome Latin America)

GAETE, N., M. CERVELLINO, y R. IRIBARREN. Proyecto $1998 \mathrm{Ms}$
Camino de acceso desde Copiapó a Mina Refugio/Compañía Minera Maricunga. Tramo Quebrada Carrizalillo. Trabajos de Mitigación de Im- pactos y de Conservación Preventivas. Comuna de Tierra Amarilla, Provincia de Copiapó, III Región de A tacama, para Compañía Minera Maricunga, 1998

INSTITUTO GEOGRAFICO MILITAR. Geografía III Re1989 gión de Atacama

IRIBARREN, J. Arqueología en el Valle de Copiapó. $1958 \quad$ Apartado de la Revista Universitaria (Universidad Católica de Chile), Año XLIII, pp. 167-195 + planos y láminas, Santiago.

KENDALL, A. Descripción e inventario de las formas 1974 arquitectónicas inca. Patrones de distribución e inferencias cronológicas. Revista del Museo Nacional de Lima XLII, pp.: 13-96, Lima.

MADRAZO, G. y M. OTTONELLO. Tipos de instalación pre1966 hispánica en la región de la puna y su borde. Monografías 1 del Museo Etnográfico Municipal de Olavarría, Edición de Homenaje al XXXVII Congreso Internacional de Americanistas.

NIEMEYER, $\mathrm{H}$. La ocupación incaica de la cuenca alta 1986 del río Copiapó (III Región de Atacama, Chile). El Imperio Inca, actualización y perspectivas por registros arqueológicos y etnohistóricos, Revista de Antropología Comechingonia. Volumen homenaje al $45^{\circ}$ Congreso Internacional de Americanistas, año 4, $\mathrm{N}^{\circ}$ especial, Julio (1985) - Diciembre (1986), pp. $165-294$

NIEMEYER.H., M. CERVELLINO y G. CASTILLO 1998 Culturas prehistóricas de Copiapó Impresos Universitaria, Santiago.

OCAMPO, C. Reconocimiento arqueológico en el área $1994 \mathrm{Ms}$ de influencia directa de Proyecto Aldebarán y sectores aledaños, dentro de la cuenca del río Nevado. Estudio ambiental preliminar, Proyecto Aldebarán, Minera Estrella de Oro Limitada. SRK (Sudamérica) S.A.

RAFFINO, R. Los Incas del Kollasuyu. Ramos Ame1981 ricana Editora, Buenos Aires.

REINHARD, J. Investigación arqueológica de la plata1991 formas inca ceremoniales en los volcanes de Copiapó y Jotabeche (Región de Atacama). Contribución Arqueológica 3: 29-55, Copiapó.

SEELENFREUND, A. Arqueología proyecto aldebarán, In$1997 \mathrm{Ms}$ forme final, elaborado por SRK (Sudamérica) S.A., para Compañía Minera Aldebarán. 
Base de datos 1. Sitios que manifiestan presencia inca

\begin{tabular}{|c|c|c|c|c|c|}
\hline \multicolumn{2}{|c|}{ IDENTIFICACION } & \multicolumn{2}{|l|}{ UBICACION } & \multicolumn{2}{|l|}{ LOCALIZACION } \\
\hline CODIGO & NOMBRE & AREA & SECTOR & UTM & ALTITUD \\
\hline 03 TA 086 & El Castaño & Río Jorquera & Confl. Q. Los Castaños & $430.553 \mathrm{E} / 6.930 .848 \mathrm{~N}$ & $2.250 \mathrm{~m} . \mathrm{snm}$ \\
\hline 03 TA 080 & La Aduana & Río Jorquera & Confl. Q. Sin nombre & $434.623 \mathrm{E} / 6.930 .791 \mathrm{~N}$ & $2.410 \mathrm{~m} . \mathrm{snm}$ \\
\hline 03 TA 077 & Los Fósiles & Río Jorquera & Río Jorquera & $436.596 \mathrm{E} / 6.930 .949 \mathrm{~N}$ & $2.492 \mathrm{~m} . \mathrm{snn}$ \\
\hline 03 TA 076 & Tambo de Pailahuén & Río Jorquera & Confl. Q. de Pailahuén & $431.533 \mathrm{E} / 6.930 .949 \mathrm{~N}$ & $2.350 \mathrm{~m} . \mathrm{snm}$ \\
\hline 03 TA 063 & Vizcachas del Jorquera & Río Jorquera & Conf1. Q. V. Del Jorquera & $444.474 \mathrm{E} / 6.933 .525 \mathrm{~N}$ & $2.703 \mathrm{~m} . \mathrm{snn}$ \\
\hline 03 TA 109 & Caserón de La Guardia & Río Figueroa & Caserón de La Guardia & $446.974 \mathrm{E} / 6.937 .484 \mathrm{~N}$ & $2.730 \mathrm{~m} . \mathrm{snm}$ \\
\hline 03 TA 118 & Las Coloradas & Río Figueroa & Vega La Colorada & $448.400 \mathrm{E} / 6.943 .850 \mathrm{~N}$ & $2.800 \mathrm{~m} . \mathrm{snm}$ \\
\hline 03 TA 059 & Junta del Turbio & Río Turbio & Confl. Río Figueroa & $445.830 \mathrm{E} / 6.935 .434 \mathrm{~N}$ & $2.577 \mathrm{~m} . \mathrm{snn}$ \\
\hline 03 TA 056 & Tambo del Turbio & Río Turbio & Río Turbio & $448.310 \mathrm{E} / 6.934 .802 \mathrm{~N}$ & $2.594 \mathrm{~m} . \mathrm{snm}$ \\
\hline 03 TA 048 & Las Minitas & Río Turbio & Conf1. Q. Sin nombre & $449.579 \mathrm{E} / 6.930 .961 \mathrm{~N}$ & $2.750 \mathrm{~m} . \mathrm{snm}$ \\
\hline 03 TA 044 & Angostura del Turbio & Río Turbio & Río Turbio & $450.217 \mathrm{E} / 6.929 .31 \mathrm{IN}$ & $2.737 \mathrm{~m} . \mathrm{snn}$ \\
\hline 03 TA 030 & Tambería de Río Turbio & Río Turbio & Confl. Q. Tambería & $454.063 \mathrm{E} / 6.919 .425 \mathrm{~N}$ & $3.070 \mathrm{~m} . \mathrm{snm}$ \\
\hline 03 TA 350 & Tambería El Cadillal & Río Turbio & Confl. Q. El Cadillal & $456.800 \mathrm{E} / 6.917 .200 \mathrm{~N}$ & $3.100 \mathrm{~m} . \mathrm{snm}$ \\
\hline 03 TA 023 & Junta del Cachitos & Río de Los Piuquenes & Confl. Q. Cachitos & $458.419 \mathrm{E} / 6.915 .721 \mathrm{~N}$ & $3.130 \mathrm{~m} . \mathrm{snn}$ \\
\hline 03 TA 351 & Quebrada La Tambería & Río Cáchitos & Confl. Q. De La Tambería & $455.700 \mathrm{E} / 6.898 .850 \mathrm{~N}$ & $3.525 \mathrm{~m} . \mathrm{snm}$ \\
\hline 03 TA 352 & Tambería Q. El Pan & Río Cachitos & Confl. Q. El Pan & $460.800 \mathrm{E} / 6.893 .500 \mathrm{~N}$ & $3.670 \mathrm{~m} . \mathrm{snn}$ \\
\hline $03 \mathrm{TA} 353$ & Paso de Peña Negra & Arroyo Peña Negra & Paso de Peña Negra & $458.600 \mathrm{E} / 6.880 .250 \mathrm{~N}$ & $4.334 \mathrm{~m} . \mathrm{snm}$ \\
\hline $03 \mathrm{TA} 021$ & Q. sangre de Toro & Río de Los Piuquenes & Confl. Q. Sangre de Toro & $459.395 \mathrm{E} / 6.914 .229 \mathrm{~N}$ & $3.090 \mathrm{~m} . \mathrm{snm}$ \\
\hline 03 TA 019 & Corral de Yañez & Río de Los Piuquenes & Llano de Yañez & $460.780 \mathrm{E} / 6.913 .402 \mathrm{~N}$ & $3.112 \mathrm{~m} . \mathrm{snm}$ \\
\hline 03 TA 013 & Pircas Negras & Río Pircas Negras & Confl. Río Nevado & $464.250 \mathrm{E} / 6.911 .100 \mathrm{~N}$ & $3.272 \mathrm{~m} . \mathrm{snm}$ \\
\hline 03 TA 354 & T. de Pircas negras & Río Pircas Negras & Confl. Q. Las Carretas & $467.500 \mathrm{E} / 6.898 .500 \mathrm{~N}$ & $3.700 \mathrm{~m} . \mathrm{snm}$ \\
\hline 03 TA 355 & Paso de Pircas Negras & Río Pircas Negras & Paso de Pircas Negras & $470.500 \mathrm{E} / 6.895 .350 \mathrm{~N}$ & 4. $100 \mathrm{~m} . \mathrm{snm}$ \\
\hline 03 TA 008 & Las Tamberías & Río Nevado & Llano de Nevado & $471.582 \mathrm{E} / 6.915 .750 \mathrm{~N}$ & $3.422 \mathrm{~m} . \mathrm{snm}$ \\
\hline 03 TA 007 & T. del Río nevado & Río Nevado & Llano de Nevado & $471.910 \mathrm{E} / 6.916 .117 \mathrm{~N}$ & $3.559 \mathrm{~m} \cdot \mathrm{snn}$ \\
\hline $03 \mathrm{TA} 006$ & Llano del Nevado & Río Nevado & Llano del Nevado & $471.756 \mathrm{E} / 6.9] 6.185 \mathrm{~N}$ & $3.605 \mathrm{~m} . \mathrm{snn}$ \\
\hline 03 TA 357 & Maranceles 2 & Río Nevado & Río Nevado & $473.300 \mathrm{E} / 6.919 .350 \mathrm{~N}$ & $3.670 \mathrm{~m} . \mathrm{snm}$ \\
\hline 03 TA 222 & Tambo del Río Nevado & Río Nevado & Río Nevado & $474.485 \mathrm{E} / 6.928 .967 \mathrm{~N}$ & $3.940 \mathrm{~m} . \mathrm{snm}$ \\
\hline 03 TA 220 & Nevado Jotabeche 1 & Río Nevado & Volcán Jotabeche & $478.360 \mathrm{E} / 6.936 .250 \mathrm{~N}$ & 5.860 m.snm \\
\hline 03 TA 356 & Volcán Copiapó & Sierra Azufre & Volcán Copiapó & $487.350 \mathrm{E} / 6.980 .200 \mathrm{~N}$ & $6.052 \mathrm{~m} . \mathrm{snm}$ \\
\hline
\end{tabular}




\begin{tabular}{|c|c|c|c|c|c|c|c|c|c|c|c|c|c|c|c|c|c|c|c|}
\hline & \multicolumn{8}{|c|}{ EMPLAZAMIENTO } & \multicolumn{5}{|c|}{$\begin{array}{c}\text { FORMAS } \\
\text { ESTRUCTURAS }\end{array}$} & \multicolumn{6}{|c|}{ ASOCIACION CON } \\
\hline $\begin{array}{l}n \\
0 \\
- \\
-\end{array}$ & 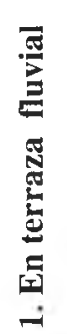 & 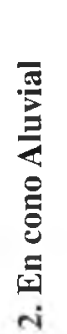 & 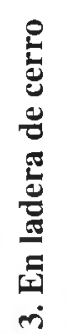 & 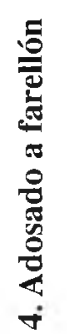 & 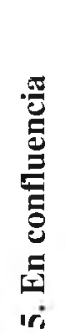 & 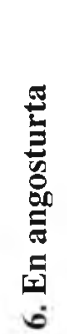 & 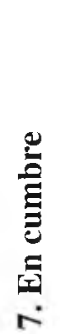 & 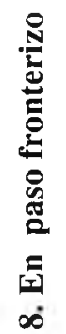 & 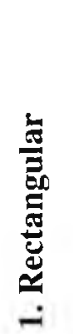 & $\begin{array}{l}\dot{j} \\
\dot{\alpha} \\
\underline{\mathbf{i}}\end{array}$ & 离 & 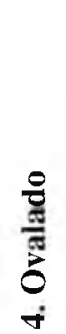 & 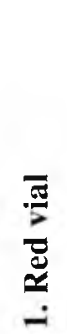 & 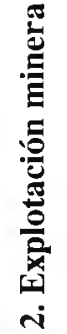 & 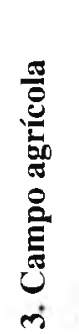 & 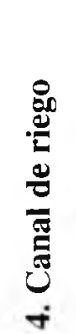 & $\underset{\text { in }}{\stackrel{\Xi}{\tilde{U}}}$ & 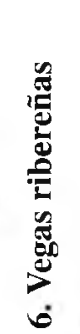 & 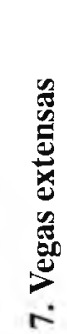 \\
\hline 03 TA 086 & $\mathrm{x}$ & 0 & 0 & 0 & $\mathrm{X}$ & $\mathrm{X}$ & 0 & 0 & 0 & $\mathrm{X}$ & $\mathrm{X}$ & 0 & $\mathrm{X}$ & $*$ & 0 & 0 & 0 & $\mathrm{x}$ & $\mathrm{X}$ \\
\hline 03 TA 080 & $\mathrm{x}$ & $\mathrm{x}$ & $\mathrm{X}$ & 0 & $\mathrm{X}$ & 0 & 0 & 0 & 0 & $\mathrm{X}$ & 0 & 0 & $\mathrm{X}$ & $*$ & $\mathrm{X}$ & $\mathrm{X}$ & $\mathrm{X}$ & $\mathrm{X}$ & 0 \\
\hline 03 TA 077 & $\mathrm{X}$ & 0 & $\mathrm{x}$ & 0 & $\mathrm{X}$ & 0 & 0 & 0 & 0 & 0 & $\mathrm{X}$ & 0 & $\mathrm{X}$ & $*$ & $\mathrm{X}$ & $\mathrm{X}$ & 0 & $\mathrm{X}$ & 0 \\
\hline 03 ТА 076 & 0 & $\mathrm{x}$ & $\mathrm{X}$ & 0 & $\mathrm{X}$ & 0 & 0 & 0 & $\mathrm{X}$ & $\mathrm{X}$ & $\mathrm{X}$ & 0 & $\mathrm{X}$ & * & 0 & 0 & 0 & $\mathrm{X}$ & 0 \\
\hline 03 TA 063 & $\mathrm{X}$ & $\mathrm{X}$ & $\mathrm{X}$ & 0 & $\mathrm{X}$ & 0 & 0 & 0 & $\mathrm{X}$ & $\mathrm{X}$ & 0 & 0 & $\mathrm{X}$ & * & $\mathrm{X}$ & $\mathrm{X}$ & 0 & $\mathrm{X}$ & $X$ \\
\hline 03 ТА 109 & 0 & 0 & 0 & $\mathrm{X}$ & 0 & $\mathrm{x}$ & 0 & 0 & $\mathrm{X}$ & 0 & 0 & 0 & $\mathrm{X}$ & 0 & 0 & 0 & 0 & 0 & $\mathrm{X}$ \\
\hline 03 TA 118 & 0 & $\mathrm{X}$ & 0 & 0 & $\mathrm{X}$ & $\mathrm{x}$ & 0 & 0 & 0 & $\mathrm{X}$ & 0 & 0 & $\mathrm{X}$ & * & 0 & 0 & 0 & $\mathrm{X}$ & $\mathrm{X}$ \\
\hline 03 TA 059 & 0 & 0 & $\mathrm{X}$ & 0 & $\mathrm{x}$ & $\mathrm{X}$ & 0 & 0 & $\mathrm{X}$ & 0 & 0 & 0 & $\mathrm{X}$ & * & 0 & 0 & 0 & 0 & 0 \\
\hline 03 TA 056 & $\mathrm{x}$ & 0 & 0 & 0 & 0 & $\mathrm{x}$ & 0 & 0 & $X$ & $X$ & 0 & 0 & $x$ & $\mathrm{X}$ & 0 & 0 & 0 & $\mathrm{X}$ & 0 \\
\hline 03 TA 048 & 0 & $\mathrm{X}$ & 0 & $\mathrm{x}$ & $\mathrm{X}$ & $\mathrm{x}$ & 0 & 0 & $\mathrm{x}$ & 0 & 0 & $\mathrm{X}$ & $*$ & $*$ & 0 & 0 & 0 & $\mathrm{X}$ & 0 \\
\hline 03 TA 044 & 0 & 0 & $\mathrm{X}$ & $\mathrm{X}$ & 0 & $\mathrm{X}$ & 0 & 0 & $\mathrm{X}$ & 0 & 0 & 0 & $X$ & * & * & 0 & 0 & $\mathrm{X}$ & 0 \\
\hline 03 TA 030 & $\mathrm{X}$ & $\mathrm{X}$ & 0 & 0 & $\mathrm{X}$ & 0 & 0 & 0 & $\mathrm{X}$ & $X$ & 0 & 0 & $*$ & * & 0 & 0 & 0 & $\mathrm{X}$ & 0 \\
\hline 03 TA 350 & $\mathrm{X}$ & 0 & 0 & 0 & $X$ & 0 & 0 & 0 & 0 & $\mathrm{X}$ & 0 & 0 & * & * & * & 0 & 0 & $*$ & 0 \\
\hline 03 TA 023 & 0 & 0 & $\mathrm{X}$ & $\mathrm{X}$ & $\mathrm{X}$ & 0 & 0 & 0 & $\mathrm{X}$ & 0 & 0 & $\mathrm{X}$ & * & * & 0 & 0 & 0 & $\mathrm{X}$ & 0 \\
\hline 03 TA 351 & 0 & $\mathrm{X}$ & 0 & 0 & $\mathrm{X}$ & 0 & 0 & 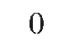 & $\mathrm{X}$ & 0 & $\mathrm{X}$ & 0 & * & * & $*$ & $*$ & $*$ & $\mathrm{X}$ & $*$ \\
\hline 03 TA 352 & $\mathrm{X}$ & $\mathrm{X}$ & $\mathrm{X}$ & 0 & $\mathrm{X}$ & 0 & 0 & 0 & 0 & $X$ & 0 & 0 & $*$ & $*$ & $*$ & $*$ & $*$ & $*$ & * \\
\hline 03 TA 353 & 0 & 0 & 0 & 0 & 0 & 0 & 0 & $\mathrm{X}$ & 0 & 0 & 0 & $\mathrm{X}$ & $\mathrm{X}$ & $*$ & 0 & 0 & 0 & 0 & 0 \\
\hline 03 TA 021 & $\mathrm{X}$ & $\mathrm{X}$ & $\mathrm{X}$ & 0 & $\mathrm{X}$ & $\mathrm{X}$ & 0 & 0 & $\mathrm{X}$ & 0 & 0 & $X$ & * & * & 0 & 0 & 0 & $\mathrm{X}$ & 0 \\
\hline 03 ТA 019 & 0 & 0 & $\mathrm{X}$ & $\mathrm{X}$ & 0 & 0 & 0 & 0 & $\mathrm{X}$ & $X$ & $X$ & 0 & $*$ & $*$ & 0 & 0 & 0 & $\mathrm{X}$ & $\mathrm{X}$ \\
\hline 03 TA 013 & 0 & 0 & $\mathrm{X}$ & 0 & $\mathrm{X}$ & 0 & 0 & 0 & $\mathrm{X}$ & 0 & 0 & $\mathrm{X}$ & * & * & 0 & 0 & 0 & $\mathrm{X}$ & $\mathrm{X}$ \\
\hline 03 TA 354 & $\mathrm{X}$ & $\mathrm{x}$ & 0 & 0 & $\mathrm{X}$ & 0 & 0 & 0 & 0 & 0 & $X$ & 0 & $*$ & * & 0 & 0 & 0 & * & * \\
\hline 03 TA 355 & 0 & 0 & $\mathrm{X}$ & 0 & 0 & 0 & 0 & $\mathrm{X}$ & $\mathrm{X}$ & 0 & 0 & 0 & $\mathrm{X}$ & * & 0 & 0 & 0 & 0 & 0 \\
\hline 03 TA 008 & $\mathrm{X}$ & 0 & 0 & 0 & $\mathrm{x}$ & 0 & 0 & 0 & 0 & 0 & 0 & $\mathrm{X}$ & $*$ & * & 0 & 0 & 0 & $\mathrm{X}$ & $X$ \\
\hline 03 TA 007 & $\mathrm{X}$ & 0 & 0 & 0 & $\mathrm{X}$ & 0 & 0 & 0 & $\mathrm{X}$ & 0 & $\mathrm{X}$ & $\mathrm{X}$ & * & * & 0 & 0 & 0 & $\mathrm{X}$ & $\mathrm{X}$ \\
\hline 03 TA 006 & 0 & $\mathrm{X}$ & $\mathrm{X}$ & 0 & $\mathrm{X}$ & 0 & 0 & 0 & 0 & 0 & $\mathrm{X}$ & $X$ & * & * & 0 & 0 & 0 & $\mathrm{X}$ & $X$ \\
\hline 03 TA 357 & $\mathrm{X}$ & 0 & $\mathrm{X}$ & 0 & 0 & 0 & 0 & 0 & $X$ & 0 & 0 & 0 & * & * & 0 & 0 & 0 & $\mathrm{X}$ & 0 \\
\hline 03 TA 222 & $\mathrm{X}$ & 0 & 0 & 0 & 0 & 0 & 0 & 0 & 0 & $\mathrm{X}$ & $X$ & 0 & $\mathrm{X}$ & * & 0 & 0 & 0 & 0 & 0 \\
\hline 03 TA 220 & 0 & 0 & 0 & 0 & 0 & 0 & $\mathrm{X}$ & 0 & $X$ & 0 & 0 & 0 & $*$ & 0 & 0 & 0 & 0 & 0 & 0 \\
\hline 03 TA 356 & 0 & 0 & 0 & 0 & 0 & 0 & $\mathrm{X}$ & 0 & $\mathrm{X}$ & 0 & 0 & 0 & * & 0 & 0 & 0 & 0 & 0 & 0 \\
\hline
\end{tabular}




\begin{tabular}{|c|c|c|c|c|c|c|c|c|c|c|c|c|c|c|c|c|c|}
\hline n & \multicolumn{5}{|c|}{ ALFARERIA } & \multicolumn{8}{|c|}{ ARTEFACTOS LITICOS } & $\begin{array}{c}\mathbf{H} \\
-\end{array}$ & \multicolumn{3}{|c|}{ ECOFACTOS } \\
\hline $\begin{array}{l}0 \\
- \\
- \\
- \\
n\end{array}$ & 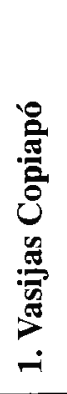 & 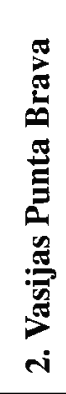 & 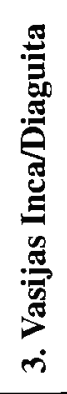 & 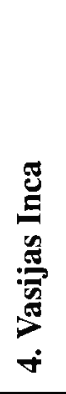 & 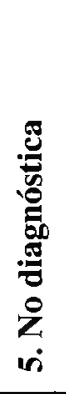 & 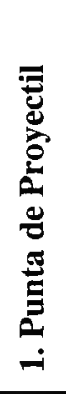 & 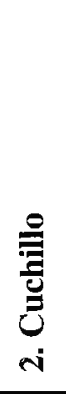 & 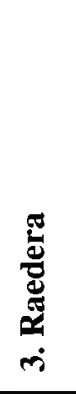 & 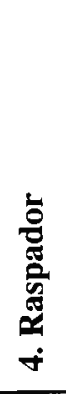 & 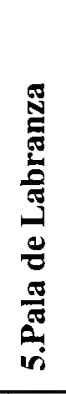 & 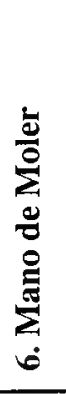 & 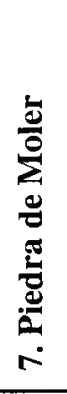 & 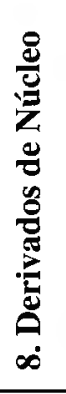 & 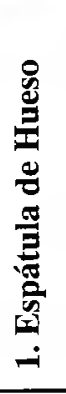 & 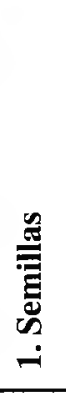 & 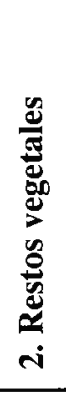 & 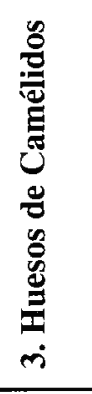 \\
\hline 03 TA 086 & $\mathrm{X}$ & $\mathrm{X}$ & $\mathrm{X}$ & $\mathrm{X}$ & $\mathrm{X}$ & $\mathrm{X}$ & $\mathrm{X}$ & $\mathrm{x}$ & $\mathrm{X}$ & 0 & $\mathrm{X}$ & $\mathrm{X}$ & $\mathrm{X}$ & $\mathrm{X}$ & $\mathrm{X}$ & $\mathrm{X}$ & $\mathrm{X}$ \\
\hline 03 TA 080 & $\mathrm{X}$ & 0 & 0 & $\mathrm{X}$ & 0 & 0 & 0 & 0 & 0 & 0 & 0 & 0 & $\mathrm{X}$ & 0 & $\mathrm{X}$ & $\mathrm{X}$ & 0 \\
\hline 03 TA 077 & $\mathrm{X}$ & $\mathrm{X}$ & $\mathrm{X}$ & 0 & $\mathrm{X}$ & $\mathrm{X}$ & 0 & 0 & 0 & 0 & $\mathrm{X}$ & $\mathrm{X}$ & $\mathrm{X}$ & $\mathrm{X}$ & $\mathrm{X}$ & $\mathrm{X}$ & $\mathrm{X}$ \\
\hline 03 TA 076 & $\mathrm{X}$ & 0 & $\mathrm{X}$ & 0 & 0 & 0 & 0 & 0 & 0 & 0 & 0 & 0 & 0 & 0 & $*$ & $*$ & $*$ \\
\hline 03 TA 063 & 0 & 0 & 0 & $\mathrm{X}$ & $\mathrm{X}$ & 0 & 0 & 0 & 0 & 0 & 0 & 0 & $\mathrm{X}$ & 0 & $\mathrm{X}$ & $\mathrm{X}$ & $\mathrm{X}$ \\
\hline 03 ТА 109 & $\mathrm{X}$ & 0 & 0 & $\mathrm{X}$ & $\mathrm{X}$ & $\mathrm{X}$ & $\mathrm{X}$ & 0 & 0 & 0 & 0 & 0 & $\mathrm{X}$ & 0 & 0 & 0 & $X$ \\
\hline 03 TA 118 & 0 & 0 & 0 & 0 & 0 & $*$ & $*$ & $*$ & $*$ & $*$ & $*$ & * & $*$ & * & * & $*$ & $*$ \\
\hline 03 TA 059 & 0 & 0 & 0 & 0 & 0 & 0 & 0 & 0 & 0 & 0 & 0 & 0 & 0 & 0 & 0 & 0 & 0 \\
\hline 03 TA 056 & $\mathrm{X}$ & 0 & 0 & 0 & $\mathrm{X}$ & 0 & 0 & 0 & 0 & 0 & 0 & 0 & $\mathrm{X}$ & 0 & * & $*$ & $*$ \\
\hline 03 TA 048 & 0 & 0 & 0 & 0 & 0 & 0 & 0 & 0 & 0 & $\mathrm{X}$ & 0 & 0 & 0 & 0 & $*$ & $*$ & $*$ \\
\hline 03 TA 044 & 0 & 0 & 0 & 0 & 0 & 0 & 0 & 0 & 0 & $\mathrm{X}$ & 0 & 0 & 0 & 0 & $*$ & $*$ & $*$ \\
\hline 03 TA 030 & $\mathrm{X}$ & 0 & $\mathrm{X}$ & 0 & 0 & 0 & 0 & 0 & 0 & 0 & 0 & 0 & 0 & 0 & $*$ & * & $*$ \\
\hline 03 TA 350 & 0 & 0 & $\mathrm{X}$ & 0 & 0 & 0 & 0 & 0 & 0 & 0 & 0 & 0 & $\mathrm{X}$ & 0 & $*$ & $*$ & $*$ \\
\hline 03 TA 023 & 0 & 0 & 0 & 0 & 0 & $\mathrm{X}$ & $\mathrm{X}$ & 0 & 0 & 0 & $\mathrm{X}$ & 0 & $\mathrm{X}$ & 0 & $*$ & * & $*$ \\
\hline 03 TA 351 & $*$ & $*$ & $*$ & $*$ & $*$ & $*$ & $*$ & $*$ & $*$ & $*$ & * & * & $\mathrm{X}$ & 0 & * & * & * \\
\hline 03 TA 352 & 0 & 0 & 0 & 0 & 0 & 0 & 0 & 0 & 0 & 0 & 0 & 0 & 0 & 0 & 0 & 0 & 0 \\
\hline 03 TA 353 & 0 & 0 & $\mathrm{X}$ & 0 & 0 & 0 & 0 & 0 & 0 & 0 & 0 & 0 & 0 & 0 & 0 & 0 & 0 \\
\hline 03 TA 021 & 0 & 0 & 0 & 0 & 0 & 0 & 0 & 0 . & 0 & 0 & 0 & 0 & 0 & 0 & 0 & 0 & 0 \\
\hline 03 TA 019 & $\mathrm{X}$ & 0 & $\mathrm{X}$ & 0 & 0 & $\mathrm{X}$ & 0 & 0 & 0 & 0 & 0 & 0 & 0 & 0 & $*$ & $*$ & $*$ \\
\hline 03 TA 013 & $\mathrm{X}$ & 0 & $\mathrm{X}$ & 0 & 0 & 0 & 0 & 0 & 0 & 0 & 0 & 0 & $\mathrm{X}$ & 0 & 0 & 0 & 0 \\
\hline 03 TA 354 & 0 & 0 & 0 & 0 & $\mathrm{X}$ & 0 & 0 & 0 & 0 & 0 & 0 & 0 & $\mathrm{X}$ & 0 & 0 & 0 & 0 \\
\hline 03 TA 355 & $\mathrm{X}$ & 0 & 0 & $\mathrm{X}$ & 0 & 0 & 0 & 0 & 0 & 0 & 0 & 0 & 0 & 0 & 0 & 0 & 0 \\
\hline 03 TA 008 & 0 & 0 & 0 & 0 & 0 & 0 & 0 & 0 & 0 & 0 & 0 & 0 & $\mathrm{X}$ & 0 & 0 & 0 & 0 \\
\hline 03 TA 007 & $\mathrm{X}$ & 0 & $\mathrm{X}$ & $\mathrm{X}$ & $\mathrm{X}$ & $\mathrm{X}$ & 0 & 0 & 0 & 0 & 0 & 0 & $\mathrm{X}$ & 0 & 0 & $\mathrm{X}$ & $X$ \\
\hline 03 TA 006 & $\mathrm{X}$ & 0 & $\mathrm{X}$ & 0 & 0 & 0 & 0 & 0 & 0 & 0 & 0 & 0 & $\mathrm{X}$ & 0 & 0 & 0 & 0 \\
\hline 03 TA 357 & $\mathrm{X}$ & 0 & 0 & $\mathrm{X}$ & $\mathrm{X}$ & $\mathrm{X}$ & $\mathrm{X}$ & 0 & $\mathrm{X}$ & 0 & 0 & $\mathrm{X}$ & $\mathrm{X}$ & 0 & 0 & 0 & 0 \\
\hline 03 TA 222 & 0 & 0 & 0 & 0 & 0 & 0 & 0 & 0 & 0 & 0 & 0 & 0 & 0 & 0 & 0 & 0 & 0 \\
\hline 03 TA 220 & 0 & 0 & 0 & 0 & 0 & 0 & 0 & 0 & 0 & 0 & 0 & 0 & 0 & 0 & $*$ & $*$ & $*$ \\
\hline 03 TA 356 & 0 & 0 & 0 & 0 & 0 & 0 & 0 & 0 & 0 & 0 & 0 & 0 & 0 & 0 & $\mathrm{X}$ & $X$ & $*$ \\
\hline
\end{tabular}

$\mathrm{X}=$ presente $/ 0=$ ausente $/ *=$ no determinado 